\title{
GMAT: The Groupwise Medial Axis Transform for Fuzzy Skeletonization and Intelligent Pruning
}

\author{
Aaron D. Ward and Ghassan Hamarneh \\ Simon Fraser University, Burnaby, BC V5A 1S6, Canada, \\ \{award, hamarneh\}@cs.sfu.ca, \\ WWW home page: http://mial.cs.sfu.ca/
}

\begin{abstract}
There is a frequent need to compute medial shape representations of each of a group of structures, e.g. for use in a medical study of anatomical shapes. We present a novel approach to skeletonization that leverages information provided from such a group. We augment the traditional medial axis transform with an additional coordinate stored at each medial locus, indicating the confidence that the branch on which that locus lies represents signal and not noise. This confidence is calculated based on the support given to that branch by corresponding branches in other skeletons in the group. We establish the aforementioned correspondence by a set of bipartite graph matchings using the Hungarian algorithm, and compute branch support based on similarity of computed geometric and topological features at each branch. This groupwise skeletonization approach supports an intelligent pruning algorithm, which we show to operate quickly and provide pruning in an intuitive manner. We show that the method is amenable to automatic detection of skeletal configurations with one, or more than one, topological class of skeletons. This is useful to medical studies which often involve patient groups whose structures may differ topologically.
\end{abstract}

\section{Introduction}

Medical research investigating the shapes of anatomical structures aims to produce visualizations and statistical outputs that help in diagnosis, prediction, evaluation of therapy, and are intuitively understood. Medial shape representations are an attractive choice for such studies because they encode object-centric, intuitively understood shape descriptors such as bending, thickness, and elongation. However, the use of medial shape representations presents a number of interesting challenges, including finding a means of stably computing them. Blum's medial axis transform (MAT) computes the centers and radii of disks lying within the object, touching at least two points on the boundary [1]. The MAT is attractive due to its simplicity and the fact that it yields a bijection between boundary and medial representations of an object. However, it is well known that the MAT is unstable with respect to small boundary perturbations; such perturbations can cause substantial changes to the topology of the MAT (illustrated in the second row of figure 1). 


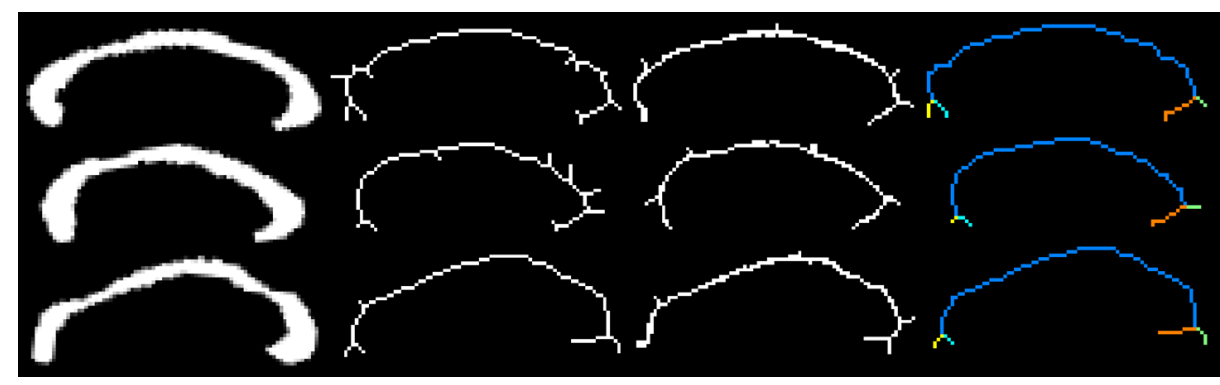

Fig. 1. Motivation for this paper. First column: 3 corpus callosum images. Second column: Conventional MAT of those images, showing many extraneous branches caused by small boundary perturbations. Third column: Results of a recent discrete curve evolution algorithm that yields pruned skeletons without incorporating group information [2]. Fourth column: Pruned, branch-corresponded skeletons given by our intelligent pruning method based on the proposed GMAT.

There exist two main approaches to this instability problem. The first is to attempt to prune extraneous branches from skeletons provided by the MAT, or to provide skeletonization algorithms which yield fewer such branches, usually by curve smoothing prior to skeletonization $[2,3]$. The second is to decide $a$ priori on the topology that skeletons should have for the class of object at hand, initialize skeletons with the chosen topology within the objects, and deform them until they lie approximately medial to the objects [4,5]. Despite substantial research in the first category, at present there exists no pruning technique that is sufficiently effective for use in medical shape studies. This is illustrated by the third column of figure 1; a leading skeleton pruning technique [2] fails to provide pruned skeletons that correspond sufficiently as to be used for a statistical shape analysis. Research in the second category has proven fruitful, but at the expense of the required a priori knowledge of the single skeleton topology suitable for all shapes in a study. This is complicated by the fact that a single such topology may not exist; in a medical study of shapes one may encounter the situation where a group of controls differs from a group of abnormal patients in terms of skeletal topology. In such cases, choosing a single topology for all skeletons restricts the study at the outset.

In this paper, we propose a skeletonization method which augments the conventional MAT by using information from a group of structures to compute a confidence value for each branch of each skeleton in the group. This confidence value represents the amount of support given to that branch by all corresponding branches in the other skeletons in the group. We call this approach the groupwise medial axis transform (GMAT). Based on the GMAT, we propose an iterative approach to intelligent skeleton pruning which removes from the group the branch with the lowest confidence value at each iteration, so that as iterations proceed, we reach the maximum common skeletal topology among all skeletons. We also describe a method for detecting potentially useful skeletal configurations involving more than one topological class, and providing samples of such configurations to the medical researcher so that he/she can make a 
choice appropriate to the study at hand. The philosophy behind this approach is that in many applications, including medical applications, multiple structures need to be skeletonized. Rather than attempting to perform skeletonization on a structure-by-structure basis or impose a fixed-topology skeleton, we leverage the information provided by the group as a whole to distinguish branches arising from true signal from those arising from boundary noise. The fourth column of figure 1 gives a preview of our results, showing a set of pruned skeletons with common topology across the group.

The remainder of this paper is organized as follows. In section 2.1 we describe the computation of the GMAT for a group of binary images, and give our algorithm for intelligent pruning based on the GMAT in section 2.2. In section 3 we show results on corpus callosum data, as well as on fish silhouettes. In section 4 we discuss our results and possible future research directions based on this work.

\section{Method}

All symbols in the text and figures of this section are conveniently defined in table 1 for reference. Figure 2 (top) provides a schematic illustration of the meanings of many variables and should be consulted in conjunction with table 1 .

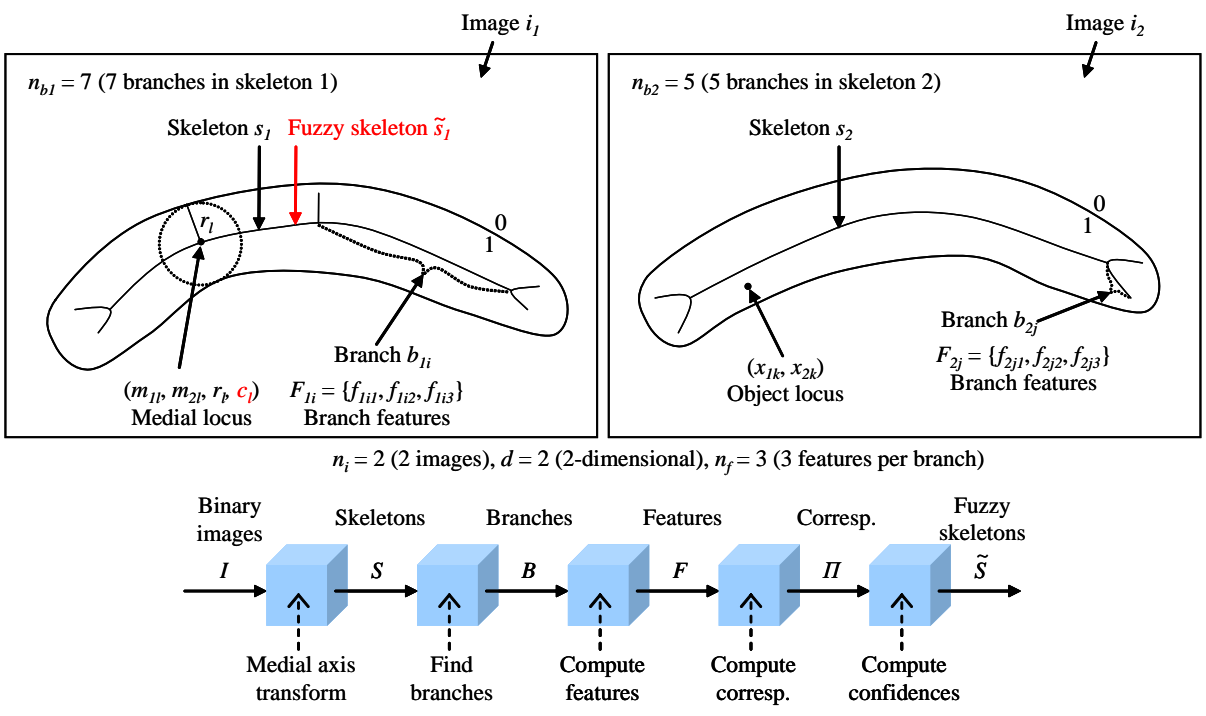

Fig. 2. Top:Illustration of the symbols used to refer to the number of binary images and skeletons in the group, their dimensionality, loci within the binary image and along the skeletons, skeletal branches, and the features (and number thereof) computed at each branch. The red symbols indicate the encoding of one of our main contributions: the fuzzification of the skeleton via a value at each branch recording the confidence that the branch contains signal and not noise. Table 1 contains definitions of all symbols used in this paper. Bottom: A block diagram depicting the steps in the GMAT. See text for details. 


\subsection{The Groupwise Medial Axis Transform (GMAT)}

The GMAT skeletonizes a group of binary images, returning a set of skeletons whose loci and radii correspond exactly to those that would be given by the conventional MAT [1]. Additionally, the GMAT provides a confidence value $0 \leq$ $c \leq 1$ at each locus. Higher confidence values associated with a skeleton branch indicate a greater probability that the branch represents signal rather than noise, as determined by the support given to that branch by corresponding branches in the remainder of the group.

The high-level process of computing the GMAT is described in figure 2 (bottom). We start with a set of binary images $I$ containing segmented and aligned structures to be skeletonized. We then perform the conventional MAT [1] on each image in the set, yielding a set of skeletons $S$. Next, we locate each of the distinct branches in each skeleton, yielding a set of branches $B$. This is done by splitting the skeleton at its junction loci. In this work, for $2 \mathrm{D}$ skeletons, these are defined as loci with a Hilditch crossing number [6] greater than 2. Next, for each branch of each skeleton, a set of scalar feature values is computed. In this paper, we compute the following six features: (1) the number of loci in the branch; (2) the $x$ coordinate and (3) the $y$ coordinate of the branch centroid, expressed as a fraction of the width and height, respectively, of the structure; (4) the sum of the radius values along the branch; (5) the angle of the branch; and (6) the number of branches neighbouring the branch. The feature values are normalized to lie in the range $[0,1]$.

The next step is to establish correspondence between each branch on each skeleton and a branch on every other skeleton, if such a corresponding branch exists. This correspondence is established based on the agreement of features between branches. This is done by solving $n_{i} \times\left(n_{i}-1\right)$ bipartite graph matching problems between all possible pairs of skeletons, where each graph is considered to have two sets of disjoint vertices. For each such matching, the first set of vertices correspond to the branches in one skeleton, and the second set of vertices to branches in another. We use the polynomial-time Hungarian algorithm [7] to solve these bipartite graph matching problems, thus computing maps $\pi_{i j}, 1 \leq$ $i \leq n_{i}, 1 \leq j \leq n_{i}, j \neq i$. Map $\pi_{i j}$ gives the correspondence of the branches of skeleton $s_{i}$ to the branches of skeleton $s_{j} . \Pi$ is the set of all of these maps.

Last, we augment the skeletons $S$ to become fuzzy skeletons $\tilde{S}$, using the set of maps $\Pi$ to compute confidence values $c$ at each skeleton locus. To compute these confidence values, we first compute for each branch an error value, which measures the mismatch between that branch's features and the features of all other branches to which that branch corresponds, according to $\Pi$. This error value is computed for all loci on branch $b_{i j}$ as

$$
\begin{gathered}
\epsilon\left(b_{i j}\right)=\sum_{l=1}^{n_{i}} \sum_{k=1, k \neq i}^{n_{i}} d_{b}\left(b_{i j}, b_{k\left[\pi_{l k}\left(\pi_{i l}(j)\right)\right]}\right), \text { where } \\
d_{b}\left(b_{p q}, b_{r s}\right)=\left\{\begin{array}{ll}
\sqrt{\sum_{k=1}^{n_{f}} d_{f k}\left(f_{p q k}, f_{r s k}\right)^{2}} & \text { if } b_{r s} \neq \emptyset \\
n_{f} & \text { otherwise }
\end{array},\right.
\end{gathered}
$$


and $d_{f k}$ is a distance function appropriate to the manifold of feature $k$. In this paper, $d_{f k}$ represents Euclidean distance for all features except for the branch angle feature, where $d_{f k}$ is 2 times the geodesic distance on the manifold $S^{1}$. Since all feature values were normalized to lie in the range $[0,1]$ prior to computing $d_{f k}$, it follows that $n_{f}$ is the maximum observable feature difference for a pair of branches. In the case where $b_{p q}$ has no such corresponding branch (i.e. $b_{r s}=\emptyset$ ), then the distance is computed to be the maximum possible error $n_{f}$. Intuitively, equation 2 computes the feature distance between corresponding branches $b_{p q}$ and $b_{r s}$ if $b_{p q}$ has a corresponding branch $s$ in skeleton $r$. We then compute the confidence value $c$ for all fuzzy skeleton loci on branch $\tilde{b}_{i j}$ as $1-\epsilon\left(b_{i j}\right) /\left(\left(n_{i}-\right.\right.$ 1) $\left.\times n_{f}\right)$. The denominator $\left(n_{i}-1\right) \times n_{f}$ used to normalize the confidence values represents the maximum possible error that can be observed for a given branch; the maximum feature error $n_{f}$ multiplied by all $n_{i}-1$ skeletons from which we attempt to find support from a matching branch.

Although a correspondence between all branches of all skeletons can be established with a set of maps $\pi_{i j}$ for a fixed $j$ and $\forall i \neq j$, the accuracy of such a correspondence is affected by the choice of $j$. Instead, we perform (in polynomial time) all possible bipartite matchings in order to accumulate as much evidence of branch confidence as possible, without regard to the choice of a "template" skeleton $j$.

\subsection{Intelligent Pruning using the GMAT}

The GMAT of a set of images yields information useful to an iterative intelligent approach to skeleton pruning. Our approach is straightforward; given a set of images $I$, the following steps are performed: (i) $\tilde{S}=G M A T(I)$, (ii) find and remove the branch from $\tilde{S}$ that (a) contains a skeletal end point (i.e. with a Hilditch crossing number [6] of 1) and (b) has the lowest confidence value of all such branches, (iii) defuzzify $\tilde{S}$ by removing its confidence values $c$, producing a new set of conventional skeletons $S$, (iv) reconstruct a new set of images $I$ from $S$, and (v) go to step i. We denote the set of fuzzy skeletons and the correspondences obtained at iteration $i$ as $\tilde{S}^{i}$ and $\Pi^{i}$, respectively. We denote the set of all confidence values in skeletons $\tilde{S}^{i}$ as $C^{i}$.

At each iteration, we compute two global confidence measures of $\tilde{S}^{i}$ as follows. For the first measure, we separate the fuzzy skeletons into different classes according to the numbers of branches in each skeleton. Then, for each class, we compute an aggregate intra-class confidence as the mean of all of the confidence values $C^{i} \forall i$ in the class. These aggregate intra-class confidences are the averaged to produce an overall intra-class confidence value $C_{i n t r a}^{i}$. The second measure is the inter-class confidence value $C_{\text {inter }}^{i}$, taken as the mean of all of the confidence values $C^{i}$. Where all $\tilde{S}^{i}$ have the same number of branches, $C_{\text {intra }}^{i}=C_{\text {inter }}^{i}$.

\section{Results}

Figure 3 (left) plots the mean inter- and intra-class confidence values for each iteration of the intelligent pruning algorithm based on the GMAT for 5 binary 


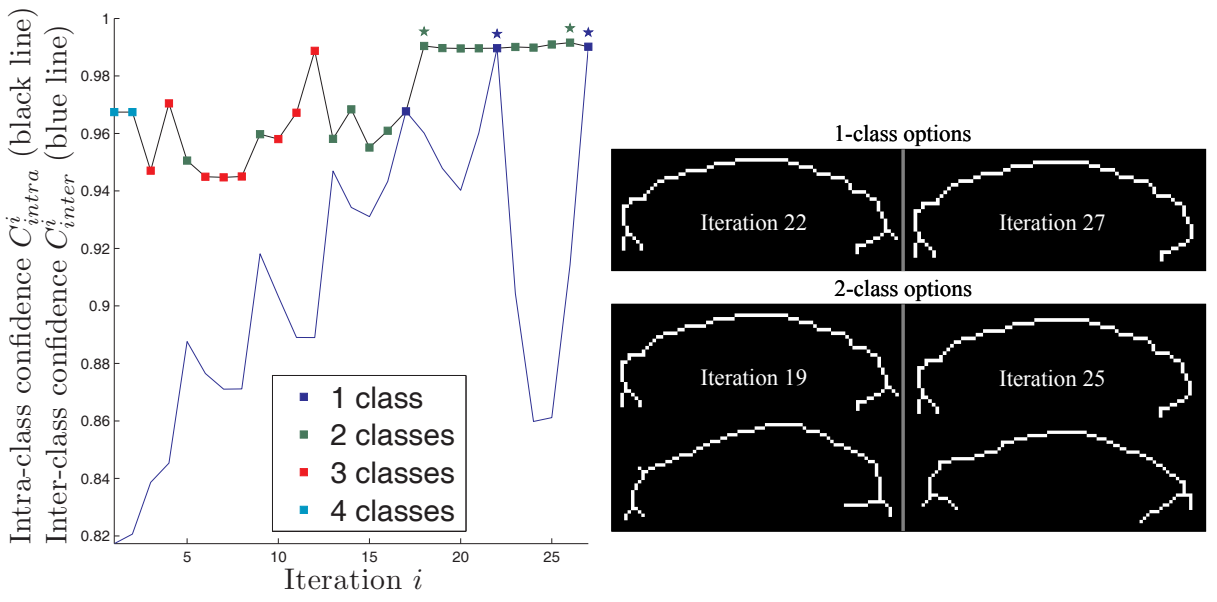

Fig. 3. Left: Mean inter- and intra-class confidence values plotted for each iteration, with plotted points coloured according to the number of skeleton classes present at that iteration. Right: The starred iterations are displayed as a menu of topological samples to aid the user in choosing a pruning iteration for his/her study. In this example the user sees a random sample from each class of skeletons at selected iterations.

images of the corpus callosum, and marks each confidence value with the number of topology classes at that iteration. Note the general tendency for $C_{\text {intra }}^{i}$ to increase as iterations proceed and skeletons evolve to become more alike, and also note that $C_{\text {intra }}^{i}=C_{\text {inter }}^{i}$ for 1-class iterations. Figure 4 shows snapshots of interesting iterations from figure 3 (left); each column shows the group of 5 skeletons. The second column (iteration 3 ) shows the elimination of red low confidence branches from the first skeleton (compare row 1, col. 1, to row 1, col. 2 ). The third column (iteration 18) shows a configuration with two topological classes (with the small orange protrusion on the left, and without). The fourth column shows a configuration with a single topological class. The fifth column shows the branch correspondence at that iteration. Iterations 1 through 22 were completed in 5 minutes and 10 seconds on a $3.0 \mathrm{GHz}$ Pentium 4 computer.

Within each contiguous set of iterations with the same number of classes (figure 3 , left), we choose the iterations with the highest value of $C_{\text {intra }}^{i}$ and provide those as choices to a user attempting to decide on a skeleton configuration appropriate to their study. Figure 3 (right) shows a prototype menu that could be displayed to a user, giving topological samples from each class at each iteration; we show only the latest two iterations in the 1- and 2-class cases due to space constraints. Once the user makes a choice, the skeletons at the corresponding iteration can be used in the shape study. We choose the highest confidence iterations within the aforementioned contiguous sets, rather than simply choosing the iterations with highest confidences overall, due to the tendency of iterations within a contiguous set to present very similar topological skeletal configurations.

Figure 5 illustrates our algorithm's ability to distinguish signal from noise in cases where very small branches represent signal and should be retained. Pictured 

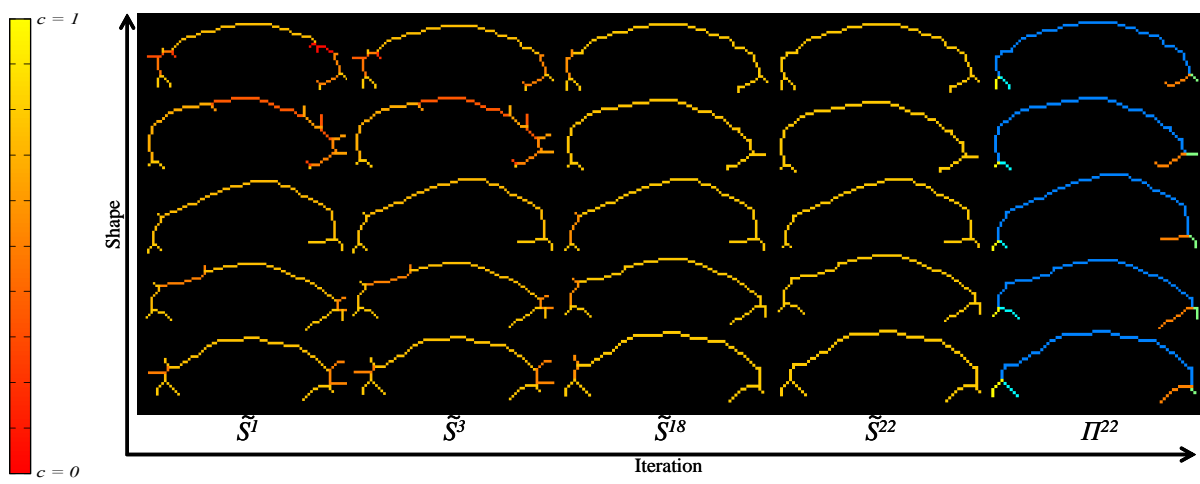

Fig. 4. This figure shows the fuzzy skeletons $\tilde{S}^{i}$ of corpus callosum images for $i=1,3,18,22$ in the first four columns (colorbar indicates map from confidence values to colors), and the correspondence between skeleton branches (same colors correspond) at iteration 22 in the fifth column.

are iterations on fish silhouettes from the Brown LEMS database [8]. The first row shows the fuzzy skeletons for the initial configurations, and the second row shows the correspondence of the pruned skeletons at the 12th iteration. Note that the very tiny branches in the red circle are retained because they have strong support from corresponding branches in the other skeletons. A skeletonby-skeleton pruning algorithm would be tempted to eliminate such branches due their very small contribution to the object's shape. Iterations 1 through 12 were completed in 4 minutes and 37 seconds on a $3.0 \mathrm{GHz}$ Pentium 4 computer.

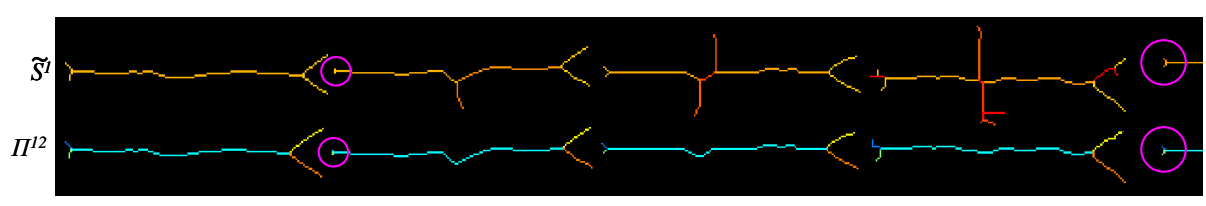

Fig. 5. This figure shows the fuzzy skeletons $\tilde{S}_{1}$ of fish images in the first row (see colorbar in figure 4) and the correspondence between skeleton branches (same colors correspond) at iteration 12 in the second row. Notice the preservation of the tiny branches (inside the purple circles, zoomed in the last column). Despite their size, they have support from the remainder of the group, so they are not pruned.

\section{Conclusions}

We have presented the groupwise medial axis transform (GMAT), and an approach to intelligent skeleton branch pruning based the GMAT. We demonstrated that pruning proceeds in an intuitive fashion based on this algorithm, and showed an approach to automatically detecting iterations containing potentially useful single- and multi-class skeletal topological configurations. To the best of our knowledge, this work is the first attempt at leveraging information 
in a group of shapes for more effective skeletonization and pruning. The value of this work is that it permits the use of medial shape representations in medical and other studies without requiring a priori selection of a single skeletal topology to be used for all shapes. We offer the user the ability to select from a visually presented set of automatically discovered, promising topological configurations; this user selection is far less demanding than requiring the user to decide on the topologies from scratch. Additionally, our algorithm is parameterless, fast, and provides useful correspondences between skeleton branches.

This work presents opportunities for many interesting avenues of future research. Our approach can be applied to 3D shapes using a different approach to splitting the 3D skeleton into its constituent surfaces. Other features than those used here can be applied; the sensitivity of the method to the choice of features will be investigated in future work. It should also be investigated whether statistical shape analysis could be performed on the skeletal configurations given by every iteration of this algorithm, delaying user involvement until the analyses are complete. This work also presents the interesting problem of performing statistical analysis of skeletons not sharing a common topology; for example where patient groups differ in terms of skeletal topology.

\section{References}

1. Blum, H.: A Transformation for Extracting New Descriptors of Shape. In: Models for the Perception of Speech and Visual Form, MIT Press (1967) 362-380

2. Bai, X., Latecki, L.J., Liu, W.Y.: Skeleton pruning by contour partitioning with discrete curve evolution. IEEE Trans. PAMI 29(3) (2007) 449-462

3. Shaken, D., Bruckstein, A.M.: Pruning medial axes. CVIU 69(2) (1998) 156-169

4. Golland, P., Grimson, W.E.L.: Fixed topology skeletons. CVPR (2000) 10-17

5. Pizer, S., Fletcher, P.T., Joshi, S., Thall, A.: Deformable M-Reps for 3D medical image segmentation. IJCV 55(2-3) (2003) 85-106

6. Hilditch, C.J.: Linear skeletons from square cupboards. In Meltzer, B., Mitchie, D., eds.: Machine Intelligence, v.4. Edinburgh University Press (1969) 403-420

7. Kuhn, H.W.: The Hungarian method for the assignment problem. Naval Research Logistics Quarterly 2 (1955) 83-97

8. Sharvit, D., Chan, J., Tek, H., Kimia, B.B.: Symmetry-based indexing of image databases. IEEE Wkshp Content-Based Access of Img \& Vid Lib (1998) 56-62 


\begin{tabular}{|c|c|}
\hline$i$ & $\begin{array}{l}\text { Image: } \mathrm{A} \text { set of } n_{p}, d \text {-dimensional object loci }\left\{\left(x_{11}, \ldots, x_{d 1}\right),\left(x_{12}, \ldots, x_{d 2}\right) \ldots\right. \\
\left.\left(x_{1 n_{p}}, \ldots, x_{d n_{p}}\right)\right\} \text { of } 1 \text { s in a binary image representing a structure, with the inside containing } \\
\text { 1s and the outside containing } 0 \text { s. }\end{array}$ \\
\hline$s$ & $\begin{array}{l}\text { Skeleton: A set of } n_{q},(d+1) \text {-dimensional medial loci }\left\{\left(m_{11}, \ldots, m_{d 1}, r_{1}\right),\left(m_{12}, \ldots,\right.\right. \\
\left.\left.m_{d 2}, r_{2}\right), \ldots,\left(m_{1 n_{q}}, \ldots, m_{d n_{q}}, r_{n_{q}}\right)\right\} \text {, where locus } j \text { specifies the center }\left(m_{1 j}, \ldots, m_{d j}\right) \text { and } \\
\left.\text { radius } r_{j} \text { of a ball of dimensionality } d \text { (the interior of a sphere of dimensionality } d-1\right) \text {. These } \\
\text { loci are taken to represent the skeleton of a binary image, such that the union of the balls } \\
\text { represented by each locus reconstructs the binary image. }\end{array}$ \\
\hline$\tilde{s}$ & $\begin{array}{l}\text { Fuzzy skeleton: A set of } n_{q},(d+2) \text {-dimensional loci }\left\{\left(m_{11}, \ldots, m_{d 1}, r_{1}, c_{1}\right) \text {, }\right. \\
\left.\left(m_{12}, \ldots, m_{d 2}, r_{2}, c_{2}\right), \ldots,\left(m_{1 n_{q}}, \ldots, m_{d n_{q}}, r_{n_{q}}, c_{n_{q}}\right)\right\} \text {, where } m \text { and } r \text { have the same mean- } \\
\text { ings as in the definition of } s \text {, and } 0 \leq c_{j} \leq 1 \text { represents the confidence that the locus } j \\
\text { represents signal and not noise. }\end{array}$ \\
\hline$b$ & $\begin{array}{l}\text { Branch: A set of } n_{r},(d+1) \text {-dimensional loci }\left\{\left(m_{11}, \ldots, m_{d 1}, r_{1}\right),\left(m_{12}, \ldots, m_{d 2}, r_{2}\right), \ldots,\right. \\
\left.\left(m_{1 n_{r}}, \ldots, m_{d n_{r}}, r_{n_{r}}\right)\right\} \text {, where } m \text { and } r \text { have the same meanings as in the definition of } s, \\
\text { specifying a branch of a skeleton. }\end{array}$ \\
\hline$\tilde{b}$ & $\begin{array}{l}\text { Fuzzy branch: A set of } n_{r},(d+2) \text {-dimensional loci }\left\{\left(m_{11}, \ldots, m_{d 1}, r_{1}, c_{1}\right) \text {, }\right. \\
\left.\left(m_{12}, \ldots, m_{d 2}, r_{2}, c_{2}\right), \ldots,\left(m_{1 n_{r}}, \ldots, m_{d n_{r}}, r_{n_{r}}, c_{n_{r}}\right)\right\} \text {, where } m, r \text { and } c \text { have the } \\
\text { same meanings as in the definition of } \tilde{s} \text {, specifying a branch of a fuzzy skeleton. }\end{array}$ \\
\hline$f$ & $\begin{array}{l}\text { Feature: A scalar value representing a feature, either geometric or topological, computed } \\
\text { based on the loci in a branch. }\end{array}$ \\
\hline$I$ & $\begin{array}{l}\text { Set of images: The set }\left\{i_{1}, i_{2}, \ldots, i_{n_{i}}\right\} \text { of binary images representing segmentations of all } \\
\text { structures in a shape study. }\end{array}$ \\
\hline$S$ & $\begin{array}{l}\text { Set of skeletons: The set }\left\{s_{1}, s_{2}, \ldots, s_{n_{i}}\right\} \text { of skeletons such that } s_{j} \text { is the medial axis trans- } \\
\text { form [1] of image } i_{j} \text {. }\end{array}$ \\
\hline$\tilde{S}$ & $\begin{array}{l}\text { Set of fuzzy skeletons: The set }\left\{\tilde{s}_{1}, \tilde{s}_{2}, \ldots, \tilde{s}_{n_{i}}\right\} \text { of fuzzy skeletons such that } \tilde{s}_{j} \text { is the } \\
\text { result of the GMAT of image } i_{j} \text {. Within this set of fuzzy skeletons, the branch(es) which most } \\
\text { certainly contain signal have a confidence value of } 1 \text { (0 for least certain branch(es)). }\end{array}$ \\
\hline$B_{i}$ & Set of branches: The set $\left\{b_{i 1}, b_{i 2}, \ldots, b_{i n_{b i}}\right\}$ of all branches in skeleton $s_{i}$ \\
\hline$\tilde{B}_{i}$ & Set of fuzzy branches: The set $\left\{\tilde{b}_{i 1}, \tilde{b}_{i 2}, \ldots, \tilde{b}_{i n_{b i}}\right\}$ of all branches in fuzzy skeletol \\
\hline$B$ & Set of branches: The set $\left\{B_{1}, B_{2}, \ldots, B_{n_{i}}\right\}$ of all branches in all skeletons in $S$. \\
\hline$\tilde{B}$ & Set of fuzzy branches: The set $\left\{\tilde{B}_{1}, \tilde{B}_{2}, \ldots, \tilde{B}_{n_{i}}\right\}$ of all branches in $\tilde{S}$. \\
\hline$F_{i j}$ & Set of features: The set $\left\{f_{i j 1}, f_{i j 2}, \ldots, f_{i j n_{f}}\right\}$ of all features computed for branch $b_{i j}$ \\
\hline$F_{i}$ & Set of features: The set $\left\{F_{i 1}, F_{i 2}, \ldots, F_{i n_{b i}}\right\}$ of all features computed for all branches $B_{i}$. \\
\hline$F$ & Set of features: The set $\left\{F_{1}, F_{2}, \ldots, F_{n_{i}}\right\}$ of all features computed for all branches in $S$. \\
\hline$\pi_{i j}$ & $\begin{array}{l}\text { Correspondence: A function } \pi_{i j}: y \rightarrow z \text { mapping each branch index } y \text { in skeleton } s_{i} \text { to a } \\
\text { branch index } z \text { in skeleton } s_{j} \text {. }\end{array}$ \\
\hline$\Pi$ & Set of correspondences: Set of functions $\left\{\pi_{i j}, 1 \leq i \leq n_{i}, 1 \leq j \leq n_{i}, i \neq j\right\}$ \\
\hline$\tilde{S}^{i}$ & Set of fuzzy skeletons: The set of fuzzy skeletons generated by pruning iteration $i$. \\
\hline$\tilde{\Pi}^{i}$ & Set of correspondences: The set of correspondences generated by pruning iteration $i$. \\
\hline$C^{i}$ & Set of confidences: The set of confidence values at all loci of $\tilde{S}_{i}$ \\
\hline$C_{\text {intra }}^{i}$ & Intra-class confidence: The mean intra-class confidence value in $\tilde{S}^{i}$. \\
\hline$C_{\text {inter }}^{i}$ & Inter-class confidence: The mean confidence value in $\tilde{S}^{i}$ \\
\hline
\end{tabular}

Table 1. Definitions of symbols used in this paper. See figure 2 for an illustration. 